\title{
Book review: \\ Quantum Scaling in Many-Body Systems, by Mucio A. Continentino
}

(World Scientific, Singapore, 2001; 188 pp.; ISBN 981-02-4389-8)

\author{
L. N. Oliveira \\ Instituto de Física de São Carlos, Universidade de São Paulo \\ Caixa Postal 369, 13560-970 São Carlos, SP, Brazil.
}

The notion of scale invariance has a long history. It permeates the work of Jonathan Swift, and was applied to fluid dynamics already in the 19th century. Thirty-five years ago, when modern theories of critical phenomena were forged, it was raised to a new status: critical exponents compared so favorably with experiments that in the theorists' eyes all previous applications of the ancient concept became pale. Viewed from the laboratory, however, comparisons that were restricted to the vicinity of critical points seemed narrow, and scaling began to be regarded as a curiosity in the junk yard of theoretical physics. Mucio Continentino changed that outlook.

In a series of papers in the late eighties and early nineties, he and his collaborators showed that scaling analyses helped identify quantum critical points in the phase diagrams of a variety of strongly correlated systems that defied the power of traditional many-body theories. Their systematic work shed light on facets that had been ignored and thus provided qualitative understanding for a number of difficult problems. He has now summarized his experience in a slender hardcover. You may well want to have this volume on your desk.

Beware the buyer, this is no primer for freshman graduate students. Even advanced doctoral candidates are likely to take in less than half of what the book is worth, because much is written between the lines. This is not to say that the text is too terse, or the algebra impenetrable.

On the contrary, the reasoning is easy to follow, and the author has been meticulous in presenting sufficient mathematical detail that a student with adequate training on Quantum and Statistical Mechanics can easily reproduce each derivation. As the experienced writer knows, however, derivations and mathematical arguments tell only half the story. The author tells the other half by means of short sentences drawing attention to the implications of the mathematical results. The best passages are brief paragraphs, loaded with physical insight, which the reader must expand in his/her mind. Carrying out such expansions is, of course, instructive, but some experience is required.

Another stimulating aspect of the work is the author's emphasis on the simplicity and generality of the scaling approach. From the outset, he makes it clear that the derivation of a scaling relation calls for a renormalizationgroup transformation. As the text evolves, he applies the same approach to different examples, to classical and to quantum models, to nearly trivial Hamiltonians and to complex ones. This pattern is a thread that binds together the twelve chapters of the book. Another thread is the author's attention to Goldstone modes. An opportunity is never missed to identify and discuss the mode associated with an application, and that is part of the richness of the book.

There is yet another unifying feature. In almost every chapter, the author has a figure, an equation, or a set of equations that epitomizes the discussion. Figure 1.2 and Eq. (2.11), for instance, summarize Chapters 1 and 2, respectively. One gets used to this pattern, and finding the epitome and seeing how it encapsulates almost every aspect of a chapter becomes one of the joys of reading the book.

The text has other virtues. Much of its strength stems from its structure. A five-chapter foundation is followed by applications, to heavy fermions, to metal-insulator transitions, to the non-linear sigma model, and to superconductors. The first part, which includes a formal introduction, discusses classical and quantum phase transitions. The treatment is standard, similar to that found in by-now classical books. Hindsight and the author's extensive first-hand experience with scaling nonetheless make those discussions remarkably rich. The only off-beat note is the chapter dedicated to real-space renormalization-group transformations. As pointed out by the author, such transformations have a simplicity that appeals to those interested in scaling techniques; in practice, however, decimation techniques have long been superseded by Steve White's Density-Matrix Renormalization-Group approach. Not to find White's work in the bibliography is disappointing.

The subsequent chapters, on heavy fermions, are excellent. Here the author combines theory and experimental data to compose what reads like a detective story. In one chapter he presents the clues: experimental data that follow the scaling law expected for dimensionality $d=0$. In the next, he solves the puzzle, explaining how this can be reconciled with the true dimensionality, $d=3$, of the heavy-fermion compounds. Once the first page of the 
former chapter is turned, expect no respite prior to reading the last page of the latter one.

Also outstanding are the three chapters on the metal-insulator transition. Concepts are adequately laid out, with emphasis on the distinction between density-driven and correlation-induced transitions. The two transitions are then discussed in detail. The chapter "Density-Driven Metal-Insulator Transitions", with its opening discussion of a non-interacting band is as enticing as it is illuminating. Figure 10.1 epitomizes the entire three-chapter unit.

The following chapter, "The Non-Linear Sigma Model", might cap the book. Combining easy-to-grasp concepts with mathematical expressions that remind the reader of those seen in previous pages, and clearly represented by Fig. 11.1, it would serve well the purpose of summarizing the text. The author, however, chose to add a twelfth chapter, 17 pages dedicated to quantum superconductor transitions. Here he has a number of interesting points to make, but the result seems poorly integrated with the preceding text. Superconductivity is a vast subject, and the author's concern with length takes a toll that detracts from the harmony of the work.

All in all, the book is nonetheless very attractive. To those who would prefer a more vigorous style, the author offers another rhythm, of long chapters that alternate with short ones, and abstract sections back-to-back with concrete examples. Those with less experience will like Continentino's unpretentious prose and his sympathy towards the reader.

Leave the book on the shelf if you lack the necessary preparation, or if you do not like condensed matter physics. Read it, otherwise. Chances are that you will be a better physicist by the time you turn the last page. A few chapters before that, you may feel the urge to fetch pencil and paper, to pursue ideas that pop in your mind. I experience that feeling, and it reminded me of a scene in Act IV of Shakespeares King Henry V, the monarch's speech to his soldiers before the battle of Agincourt: of this speech it is said that, when the monologue is finished, the audience is ready to leave the theater and join the army. Continentino is not Shakespeare, but his writing is inspiring. And is it not inspiration what we expect to find between the covers of a good book? 